\title{
Universics - A Common Formalization Framework for Brain Informatics and Semantic Web
}

\author{
Ioachim Drugus \\ Moldova Academy of Sciences, Semantic Soft, Inc \\ Republic of Moldova
}

\section{Introduction}

This chapter is an introduction to a conceptual framewok and a mathematical apparatus, said to be Universics, designed to serve for integration of other disciplines via their universes of discourse and, in particular, for the integration of Brain Informatics and Semantic Web, which are in the focus of this chapter. By universe I refer not only to what is said to be a "universe of discourse“, but also to any modeling framework where, for modeling, we use representation rather than discourse, so that such universe can be said to be a "universe of representation". Universics is a fundamental theory which posits the notion of universe (or world) with a plural universes (or worlds) in the basis of a conceptual framework, and expresses other notions through this basis.

An object said to be a universe must be rather complex and only a complex mathematical structure can serve as its formalization. I have previously outlined (Drugus, 2007) an approach to the content of mind said to be the A3 approach. This approach is introduced in this paper in full detail and used for the formalization of the concepts of Universics which, thus, becomes centered around the A3 approach. The A3 approach can also serve for the formal specification of an intelligent agent - a virtual machine for processing , mind content' which adds non-determinacy to the Turing machine to enhance its algorithmic capabilities with features of intelligence. I will outline the features of such a machine, which I say to be a conceptor, but a complete specification of conceptor would require a separate publication. I had to make reference to this intelligent agent here, because its involvement simplifies the account of Universics strongly centered around the data model of ,mind content' of such an intelligent agent.

Since the ,mind content' of an intelligent agent is obtained in result of cognition of the Universe and is "in the image " of the Universe, it is natural to say a piece of mind content to be a universe - an "inner universe ", "subjective universe", or ",subject universe“, as opposed to the "objective universe" or "object universe". Moreover, due to the A3 approach, the "mind content' has a strictly specified "format", defined in the language of an enlarged set theory said to be A3 language. Therefore, by applying "conceptual inverse engineering" (my term), I ascribe this "format" to any universe. Having this manner obtained a uniform 
formalization of the universes of discourse, by the application of other formal methods, we can look for relationships between different disciplines, discover convergence, or develop integrative methods. Since Universics' terminology and conceptuality proceeds from the largest "whole", the Universe, Universics is a holistic discipline, and since it can be used as a formal framework to discuss about other disciplines, it is a meta-discipline.

The A3 approach, its A3 language, and its A3 data model are strongly correlated with Semantic Web and its languages for knowledge representation, in particular, with the most complete one, the formal language of the OWL standard. A piece of mind content is formatted according the A3 language, and can be uniformely mapped into an OWL ontology, and viceversa.

The A3 approach makes abstraction from presentation details of Semantic Web standards, in particular, from the URI's and other web-related notions and entities, and therefore, despite its larger expression power, the A3 language is simpler than the Semantic Web languages. Also, the A3 approach complements the Semantic Web with notions and methods which simplify ontology engineering and helps in modeling the reality due to new notions, which have well established meaning in practice. Finally, the A3 approach does not need separate treatment of precise semantics in the language of set theory as all the standards of Semantic Web do, because A3 language itself is a language of an extended set theory and can be used to describe the semantics of Semantic Web standards. Finally, as a formal language, A3 is a very simple "language of brackets" (three pairs of brackets) to which a special meaning is assigned and for which special manipulation rules are formulated to obtain a strict calculus directly correlated with the $\lambda$-conversion calculus (Curry, 1941) and the Turing machine (Turing, 1937).

The idea of Universics as a mathematical holistic discipline goes back to my doctoral dissertation on superintuitionistic logics (Drugus, 1982), where I have applied a model theory strongly based on Leibniz ,possible worlds' apparatus. By that time, mathematicians, including myself, became aware that the "forcing method' used to solve the "Continuum Hypothesis“" problem (Cohen 1963-64), i.e. the problem N1 in Hilbert's collection of open problems for the 20th century, is essentially the ,possible worlds' conceptuality but is formulated in different terms. The solution of this problem is currently treated by many authors as the last strong result in the set theoretic foundation of mathematics. It became obvious that, by treating ,possible worlds' as multiple universes of discourse for set theory, a more expressive set theory can be obtained. For this purpose such universes must be formalized by moving the discourse from sets to classes and, finally, to universes of discourse, and treat the universes of discourse as entities studied by a meta-theory with respect to set theory. Universics turns out to be such a meta-theory.

In 2007 I learnt about Semantic Web and discovered that "formally" it is close to what I have been thinking twenty years before, except that Semantic Web standards add technical matter specific to the web. Also, I have soon got the conviction that wide deployment of Semantic Web is hindered by lack of "pure mathematics" for presentation methods as opposed to logical methods (of discourse) prepared for Semantic Web by the Description Logic developed in late 1990s. Finally, Semantic Web lacks a formally defined „semantic machine“ or "intelligent agent" to process the ontologies. My paper of 2007 (Drugus, 2007) is basically a general plan of development of Brain Informatics and Semantic Web based on pure mathematics and this chapter is a step in implementation of this plan. 
I also believe that the failures of the technological solutions, like those which caused the so called ,AI winter' and currently hinder the wide deployment of the Semantic Web, are due to pure engineering approaches, and such difficulties can be avoided if mathematical methods prior to engineering concrete solutions are developed. I think, that a "brain mathematics" should and can be obtained by extending the set theory, a commonly accepted foundation of mathematics, up to a theory, the entities of which have an intuitive and natural meaning for intelligence. In particular, an intelligent agent must be treated as a virtual machine which processes the sets and other entities of such an extended set theory. The results of this research are currently being implemented in software tools for Semantic Web at the Semantic Soft, Inc. company.

\section{The $A 3$ approach to brain informatics}

Universics is centered around the A3 approach which serves as its methodology and formalization framework. The A3 approach is a conceptuality and a data model said to be the A3 data model, a language said to be the A3 language, and a vision on the mechanism of mind from which all other aspects of this approach can be inferred. The A3 data model is the data model of the mind content, and it can also serve as an alternative framework for knowledge representation and formal representation of linguistic corpora (Drugus, 2009). This section is an introduction to the A3 vision of mind which discovers the main operations of mind which I would attribute to "brain mathematics".

\subsection{The A3 vision on the mechanism of mind}

The A3 approach proceeds from a vision on the process of formation of mind content, treated as a network consisting of mental entities. Also, the formation of this network is treated as "weaving" rather than "creation of a whole" because this process is perpetual and it never ends during the lifetime of mind. Since the term "formation" is too general to reflect the specifics of this process, and "weaving" is not a convenient word to serve as a term, I will use the term "synthesis" for such formation of mind content. Currently, there exist a large number of different types of networks used in research and technology - neural networks, semantic networks, Petri nets, and other types. Mind content is a new type of network.

Synthesis unfolds in steps and for a natural (i.e. not "artificial“") mind, probably, a large number of mental entities is created at one step. In order to simplify the analysis, in this paper I will limit to only one atomic mental entity created at one step of the mind content synthesis.

Since the mind content synthesis is regarded as perpetual and never ending during the lifetime of mind, it always encreases in size and, even though the mental entities are more abstract than sensual data, the mind content is always a realistic "film of the reality" - a "conceptual film". I am not interested here in the dynamic aspect of mind content which determined me to assimilate it with a "conceptual film". The dynamic aspect only shows that mind content is an "open world“, i.e. a changing object. I am interested in mind content as a "history" or "memory" of the synthesis process reflecting the "structure" or "form" of the mind content. The Figure 1 below illustrates the "mathematics of brain" and serves as a roadmap to further material. 


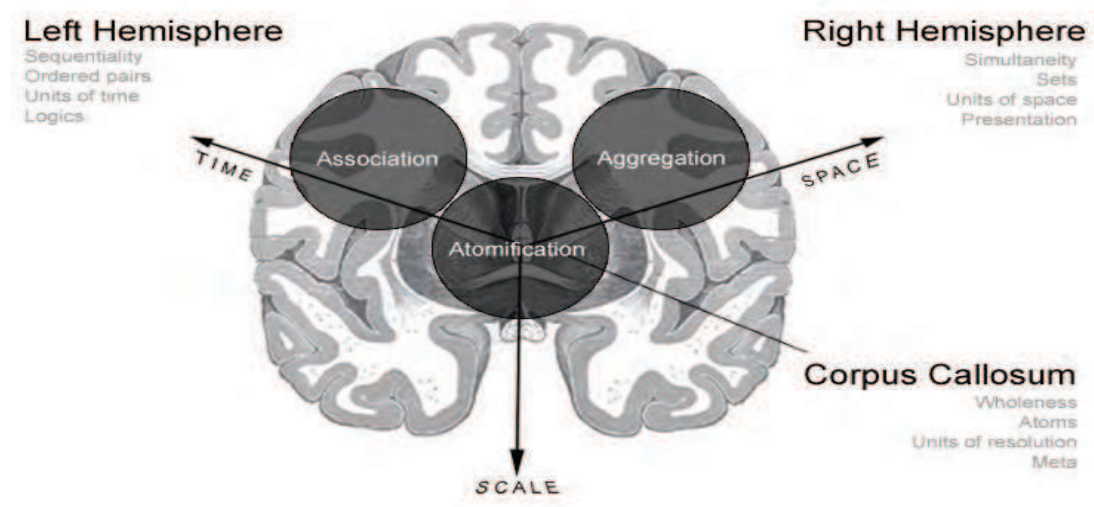

Fig. 1. Brain Mathematics

Earlier (Drugus, 2007), I treated the synthesis of mind content without the part-whole aspect. I explained that such synthesis is done by alternative involvement of each of the two hemispheres of brain at one step of synthesis, and with each involvement, it does exactly one operation whereby it creates an entity. For right-handed people, I substanciated that the left hemisphere is specialized on the association operation whereby it produces a new entity also called association, while the right hemisphere is specialized on the aggregation operation whereby it produces a new entity also called aggregation. In this paper, I will additionally treat the part-whole aspect, which became possible due to finding a better explaination of the part-whole aspect, dealt with by the third component of brain - the bridge between two hemispheres called corpus callosum. I assume this component to be specialized on the operation of atomification of the A3 approach.

The mind content obtained in result of a perpetual synthesis can serve only as a basis for intelligence, which alongside accumulation of conceptual experience as mind content, manifests via other activities, including the logical activity. But the mind content synthesis unfolds on a level which serves as a basis for higher levels of intelligence. The level immediately above it allows to cut on this wealth of conceptual data and can be said to be the level of identification. The identification allows to regard many mental entities as "the same". Because these two layers are sufficient for the discipline of Universics, in this paper, I will focus only on the level of mind content synthesis and on the level of identification.

To summarize the account of the A3 approach vision on brain mathematics, I will say, that at one step of mind content synthesis, the brain applies exactly one of the three operations, said to be $A 3$ operations, for creating a new entity:

- Aggregation operation for creating an entity also called aggregation - an entity type generalizing the notion of set of set theory, and the notions of class, container and collection of Semantic Web,

- Association operation for creating an entity also called association - an entity type generalizing the notion of ordered pair of set theory, and the notion of property of Semantic Web,

- Atomification operation for creating an entity also called atomification - an entity type generalizing the notion of atom (also said to be ur-element) of set theory and 
the notion of ,whole' of mereology, science of "parthood" or of "part-whole“ relationship (and for which there is no corresponding notion in Semantic Web).

To clarify the above, it should be added that the entities are either untyped or typed, and if an entity is typed, then it can have one of the three types above, which I will refer to as the $A 3$ types. Each of the terms aggregation, association, atomification, will serve as a name both for the operation and for its result, and we will distinguish between them by specifying to which of the two we refer. So, we will use expressions like „an aggregation“, "entity of type aggregation", or "aggregation operation" and similar expressions for "association" and "atomification".

In the most general case, I am treating the term operation as a many-to-many correspondence. So, even though A3 operations are not yet completely defined, they should be treated as many-to-many correspondences or, how they are called elsewhere - "manyvalued functions". The notion of property in Semantic Web corresponds to the notion of set theoretic relationship and of "functional property" as it is said to be there. This is why to avoid term collision, I will not say a many-to-many correspondence to be a function - I will say it to be operation.

One of the greatest difficulties in guessing how the mind content is synthesized is to find out how the representations of different objects can be retrieved at a later time. This is a general problem for any kind of networks. Also in philosophy the process of discriminating separate entities in the "material network" of the Universe has been a problem since ancient times - a problem, which has not been solved up to date.

I would have preferred the term "domain of integrity " for the representation of an object in the network of mind content, because this would have better reflected the idea of dealing with the "wholeness" treated here as a synonym for „integrity“. But „integrity“ has a special meaning in English, and I will use the term ",integral domain“, which though is good due to being shorter. I consider the integral domains, as representations of objects, to appear in mind due to the atomification operation.

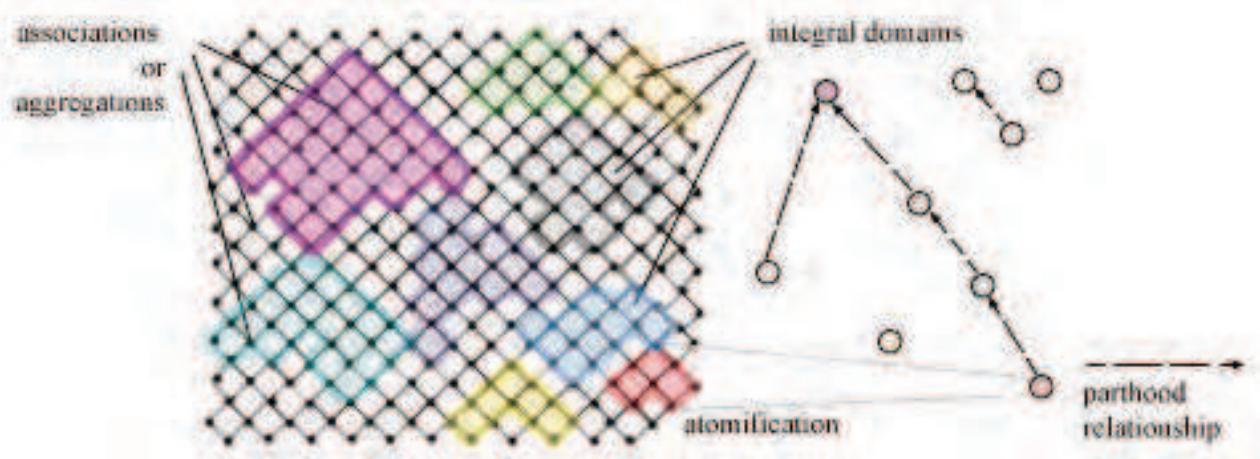

Fig. 2. A piece of mind content

The figure 2 above illustrates the mind content obtained in result of a synthesis process, which is a directed process: in this figure, we consider that mind content "grows" upwards. The results of applications of both the aggregation and association operation are represented as nodes of the network. In order to simplify the picture, the distinction between the entity types aggregation and association is not indicated in the figure and all the aggregations in 
this figure are deemed to be two-element aggregations - same number of arguments as of the association operation. The colored areas are said to be integral domains. These pieces of mind content are "production" of the atomification operation, which can be thought to act in a dimension, different from the dimensions where act association and aggregation. The results of applications of the atomification operation can be imagined to lie in space above the $2 \mathrm{D}$ space of the picture. This is why, to represent the integral domains as new entities not lying in the plane and to be able to discriminate between them, they are represented in different colors. The bottom of each integral domain is represented by the atoms (indivisible constituents) of the represented object. This botom is "dented" because in the synthesis process the representations of the atoms appear at different times. The top of each integral domain is represented by exactly one entity which we regard as the unity of this integral domain. The first intuitive idea about the manner how an integral domain can be extracted from the mind content is by imagining that such a domain is "carved out" from the mind content taking into account the two boundaries - top boundary represented only by the unity, and bottom boundary represented by many atoms.

Notice that in the Universe of matter, one body B is part of another body C, if B is spatially "inside" C. The universe of mind differs from the Universe of matter and the representations of $B$ and $C$ in mind look totally different. Namely, the unity of B becomes an atom of $C$, so that in a graphical representation as that of Figure 2, the representation of B would be beneath (and not inside) the representation of $\mathrm{C}$.

\subsection{Place of A3 approach among other approaches}

In order to correctly place the A3 approach among others, I will mention the features of three main existing approaches and explain why they are incomplete for knowledge representation purposes and for describing the mind content.

The relational model (Codd, 1970) was placed in the basis of database technology and the entity-relationship approach (Chen, 1976) is successfully used in object management and UML. Some authors regard a, world' as represented by a database where all data about the entities of such a world are persisted. Both these two main modeling frameworks, widely used today in IT and AI implementations, don't offer the level of precision required by mathematics. Really, set theory is regarded as the main formalization framework for mathematics and other disciplines, but the notion of set and ordered pair (treated as „element of order" ), not only are not among the primitive notions of these approaches, but are used uncounsciously. Set theorists know that such attitude to the intuitive notion of set can raise serious logical contradictions. In software, logical contradictions manifest as bugs. Therefore, the software for representation of mind content developed according approaches which unconsciously treat the basic operations of mind will, probably, have "conceptual bugs" in their specification and will not work.

The sound mathematical foundation of Semantic Web is reflected in its standards by formulation of semantics of standards in the language of set theory. But Semantic Web is focused on the discourse about the Universe, rather than the representation of the Universe. The representation capabilities are an essential feature of an intelligent agent, and a representation framework missing in Semantic Web might be responsible for the fact that, so far, there is no proposal of a generic agent for processing the Semantic Web data.

Also, even though the notion of class, which is a conceptual conterpart of the notion of set, is among its primitive notions, Semantic Web standards totally ignore the notion of atom, or 
"urelement" of set theory, and this results in difficulties to strictly discriminate between the notions of individual and of class. Say, according OWL, an individual can also be a class. But then, it remains unclear what is an individual which is not a class and how to express in OWL the property of such an entity. Despite that the notion of class is examined by Semantic Web, and the other notions correlated with class, like container and collection, are also used all such notions are regarded as independent of each other and no correlation between them is indicated. Finally, the SPARQL querry language uses graphs to indicate different universes of discourse without any indication of correlation of such graphs with the notions class, container and collection.

All the difficulties above show that Semantic Web, as it is currently formulated via different standards, also does not completely satisfy the requirements for a really mathematical approach. True, we can clearly separate the aspect which Semantic Web currently does not cover - this is presentation of entities, versus discourse about entities. Even after such separation, there remains the main difficulty on the path of wide deployment of Semantic Web to become a really "democratic" tool - the extreme complexity of the standards, which shows that there is insufficient mathematics behind them.

\subsection{The operational and structural completeness of the A3 approach}

The activity of an agent consists of separate operations, and the agent builds data structures also by applying various operations. I am treating an approach to intelligence as mathematical, if it

(1) Specifies a set $\mathrm{S}$ of mathematical operations which our mind is capable to do,

(2) Substanciates that other operations of mind can be reduced to the operations in $S$ (operational completeness),

(3) Substanciates that any structure represented as mind content can be represented as a result of multiple of applications of these operations (structural completeness).

I will say a set $S$ of mathematical operations to be an orthogonal basis of a class $C$ of operatons if additionally to the conditions above, the operations in $S$ are irreducible to one another. Can there exist such a set $S$ of operations in natural intelligence?

Since a brain neuron can do a limited number of operations and these are sufficient for the neuron to serve as a building block for the physical substratum of intelligence, there must exist a limited number of operations to which all phenomena of logical mind can be reduced. This shows that the operational-structural mechanism of the brain and the "soft" layer above it, the mind, must have an orthogonal basis of operations. But, since we are talking about the mind content, such an orthogonal basis should be looked for in the mind and there is only one method to study the mind - introspection.

The A3 operations are an orthogonal basis of operations of mind defined above. This basis was found by introspection and this fact is substanciated by reference to neuro-science, physics, and set theory. The A3 approach is a mathematical approach, because the A3 operations are mathematical operations - they are generalizations of set theoretic operations. The thesis that the A3 operations are operationally and structurally complete, cannot be proven and its validity is a matter of belief. This thesis has the same status as Church-Turing thesis about the algorithmic completeness of the programs for Turing machines, and I will refer to this thesis as the $A 3$ completeness thesis. 


\section{Conceptor - an intelligent agent for the Universics}

The A3 approach is an approach to intelligence, which proceeds from a generic intelligent agent capable to create entities of the $\mathrm{A} 3$ types by applying the A3 operations and process the structures created this manner said to be mind content. The Latin for "to create an entity" in mind is "concipere" - a word from which comes the family of words correlated with "concept". Therefore, by analogy with the commonly used in Pattern Recognition term ,perceptron', but without mixing Latin (percepere) with Greek (-tron), I introduce the term conceptor. I will say a conceptor to be an agent capable of two activities:

- Creating structures by multiple applications of A3 operations, and

- Using such structures

According the A3 approach vision on the mechanism of mind, the brain is a conceptor. The characteristics of conceptor among other possible intelligent agents, is that the conceptor is meant to be capable to do "brain mathematics" - i.e. create mind content by applying mathematical operations, which in our case are chosen to be the A3 operations. I regard a conceptor as a "lower level“ mechanism upon which builds the "upper level“ mechanism of intelligence, and which, in order to stick to mathematical methods, can be said to be a mechanism of reduction of any operation to multiple applications of the A3 operations. Obviously, the use of the term "mind content" for a conceptor imposes to also use the term "mind" for a conceptor. Also, I will use in discourse about conceptors the other terms previously used in discourse about human brains.

All "superior" animals have a two hemispheres brain with a bridge between them, which means that the Nature, as the best tester of mechanisms of life, found out that specialization is essential for intelligence. Thus, even though according some views the inanimate matter can bear features of intelligence so that the whole Universe can be regarded as intelligent, I would regard specialization in doing main mathematical operations as a distinguishing feature of intelligence. Therefore, as the first requirement in specification of a conceptor, I regard its full compliance with the A3 vision on brain - namely, a conceptor must have three components each specialized on one of the A3 operations. Going forward, I will also use for the components of a conceptor same names as for the human brain - "hemisphere", "left hemisphere", "right hemisphere". This cannot raise confusion within the context of the A3 approach, because "operationally", i.e. with respect to the A3 operations, the components of a conceptor and of the brain are identical as per the specification of the conceptor.

This paper is focused on how the conceptor, by applying the A3 operations, creates structures called mind content. But to get a better understanding of this aspect of the activity of a conceptor, I will also give below a general idea of how such structures are used by a conceptor in reasoning.

Prior to other intelligent activities, the conceptor must be able to view pieces of data, including pieces of mind content for the introspection processes. For this purpose it must have an "attention" focus, which can

1. Enlarge (narrow) from one aggregation to a larger (smaller) aggregation,

2. Move allong associations in both directions,

3. Zoom in from an atom representing the identity of a structure to the structure itself and viceversa.

The read-write head of a Turing machine is an example of such a focus, which though, is limited to motion along associations of a "linear universe“. By specifying for the conceptor the read-write operations similar to those of the Turing machine and introducing the states, 
we obtain a more general agent, with non-deterministic "programs", versus the deterministic programs for a Turing machine (Turing, 1936).

The conceptor is significantly richer in its behavior, including, due to being capable of doing more complex motions of its attention scope, than the Turing machine head. At the same time, all such motions are required, in order to be able to travel in a universe with all three possible axes, like the universe of mind. Conceptor is meant to serve as a conceptual virtual machine for processing data modeling mind content.

A formal specification of the conceptor as virtual machine is not the focus of this paper, but the described features are sufficient for understanding the focus of this paper - creating mind content. The material below also provides details of the conceptor's behavior, which will be included in its formal specificaton.

\subsection{The intelligence of a conceptor}

"What is intelligence?" is a question which has been extensively discussed about and the only good and widely accepted approach to anwering this question turns out to remain the "Turing test", which actually does not answer this question, but gives a clue how to compare artificial intelligence with human intelligence. Such an approach to answering this question suggests that an artificial intellect must be able to do same operations as a natural intellect. In my approach I regard a conceptor as intelligent if it, simply, repeats the functionality of brain regarding the A3 operations.

Despite that due to such simulation of brain, we might admit that an "advanced“ conceptor might have same level of intelligence as the Human, this cannot ensure higher intelligence, because we actually don't know what is intelligence. With all fuzziness of terms, we can say that intelligence is capability to cognize the Universe. An intellect is part of the Universe and, thus, intelligence is also the capability to cognize himself.

I regard the Universe as having 3 main dimensionalities - space, time and scale. Even though space, as one of such axes, in turn, can be three-dimensional as in the case of the physical Universe, this is unessential for our approach. Notice, that also in the fundamental equations of physics space is represented by one parameter, sometimes denoted by one variable " $x "$. Below, I will relate intelligence with capabilities of the brain or of a conceptor to work with these "universal“ axes.

\subsection{A conceptor's hemispheres specialization in cognition of the Universe}

I assume that the right hemisphere is specialized on processing simultaneity, i.e. it "takes shots" of simultaneously viewed entities. Same speciality must have one hemisphere of a conceptor. To view several entities "simultaneously", the entities must be present "at the same time“ and I treat the "same-timeness" as a property of space, which allows to distinguish it from its opposite - time. Thus, we can regard the right hemisphere as a virtual "space machine“ - a machine for processing spacial relationships.

I assume that the specialty of the left hemisphere is processing the sequentially viewed entities. Sequentiality in viewing is imposed by time and is a property of time which distinguishes time from space. Really, no matter how many directions has a space, and how the direction from one entity to another entity is indicated, there is a possibility to view the entities in the opposite direction. For example, given an ordered pair $(a, b)$, which is written 
in the linear space of text, we actually don't know which element is considered first and which should be regarded as second, unless we know the consensus on "viewing direction". While a piece of mind content is static, perception of a varying "content" in the Universe, like a vision or sound which change, is a dynamic process involving sequentiality in processing. Sequential order is represented in mathematics by a set of ordered pairs and an ordered pair is an association. Thus, the left hemisphere specialized on creating and processing associations can be said to be a "time machine“ - a machine for processing termporal relationships.

In the fundamental equations of physics expressed in space $x$ and time $t$ parameters, you can exchange the places of $x$ and $t$, and the equation remains valid. Due to such space-time symmetry, there is no physical law which would help distinguish between space and time. I am resolving this difficulty by considering time as a dimensionality of a universe, which allows viewing in only one direction, and space - as a dimentionality of a universe, which allows viewing in any direction.

I assume that the specialty of the bridge between hemispheres is processing the scale dimensionality and it can be said to be a "scale machine“. Scale refers to the part-whole relationship and seems to be the least studied dimensionality of the Universe. The dimensions and qualities of physical bodies relate to different units of measure, and this also relate to scale. A conceptor processes scale by the zoom in and zoom out capabilities of the attention focus. Probably, the operation of this component of a conceptor, will be better understood after we will have dealt below with atomifications.

\subsection{Identities - the units of mind content}

The main aspect of cognition is identification and for a conceptor to identify an object, it first creates an entity in mind which we will say to be the object's identity. In A3 approach we will say all the atomic (indivisible) mental entities, out of which the mind content is made, to be identities. Even though an atomic mental entity may not yet have been used as an identity of an entity at a moment of time, it is destined to serve as such at a later time - this is the explaination of this use of the term identity. With this understanding of the notion of identity, we can say that the distinguishing property of the identities among other entities is their residence in mind.

Because the Universe must have an unlimited number of objects, the next requirement to a conceptor is the capability to create identities without any limitation. An important aspect of the synthesis process is that at each step a new identity is created - notice, that in defining the vision on workings of mind we underlined the word "new". To ensure this, the conceptor must have an identity generator for creation of new identities without any limit. In the process of synthesizing mind content, the re-use of an old identity is not acceptible. This is because similar to the human mind, the conceptor must treat each situation as totally new, and only later consider different entities "the same“.

According the A3 vision on mind, the perpetural synthesis process imposes creation of many identities for one single object. This is not a peculiarity of the synthesis process but reflects the cognition process. An object in the Universe can be perceived at different moments or in different places. We will say each of such "object instance“ to be an object's presentation. The multitude of identities of an object is actually the class of identities of the object's presentations. 


\subsection{Identification and reification}

The synthesis of mind content is acompanied by a process which makes several mental entities "the same" or "identical“. This process complies with Occum's razor principle "entities should not be multipied without necessity" and is part of the "economy of mind". "To be identical" can be also treated as "having same identity" (in mind).

I will distinguish between the notion of entity and the notion of object. By entity we generally mean anything, whether in the material Universe or within the mind. By object we don't mean everything, but only an entity $\mathbf{e}$ for which there is a representation $\mathbf{r}$ in mind. So called things-in-themselves, invented by the philosopher Immanuel Kant, are entities, but they are not objects. Also, for a device recognizing pictures by a pattern, a picture which cannot be recognized because it lacks a pattern in the database of patterns, is an entity, but it cannot be said to be an object.

An object may have many identities. For example, the star Venus, also called by two other names „Morning Star" and „Evening Star", must be expected to have at least three identities in the mind of an English speaking child, until he or she learnt that this is the same object. The process which takes place when he learnt this fact is said to be identification. This process is complex and we will not study it here, but will focus only on the result of this process, which is said to be "identical entities" or "entities which are the same“. In mathematics, the fact that two entities denoted by $\mathbf{E}$ and $\mathbf{F}$ are identical is denoted by the equality symbol between them, $\mathbf{E}=\mathbf{F}$. If we denote the predicate "is identical" by the term sameAs of the OWL language, then we can denote such fact by [E sameAs F]. The use of square brackets for quotation of an expression is rather a new practice, but it makes part of the A3 language, and I will explain this type of denotation later.

For an entity denoted by e, I will denote by $=(\mathbf{e})$ or $=\mathbf{e}$ its identity. The meaning of this denotation is "neglecting the name and keeping only identity of the value", or "making the name replacible by any other name with same value". Obviously, the denotation " $=\mathrm{e}$ " is equivalent to the denotation "_: $\mathrm{e}$ " of a blank node. This is a new type of notation which treats the equality sign as denotation of a unary operator instead of a binary operator. Obviously, $\mathbf{e}$ and $=\mathbf{e}$ are equal, and we can denote this fact by $(\mathbf{e}=\mathbf{=})$ or $(=\mathbf{e}=\mathbf{e})$. We said that an entity may have many identities - which of these identities is denoted by the expression "= $\mathbf{e}^{\mu}$ ? Any identity of $\mathbf{e}$ can be also denoted by " $=\mathbf{e}^{\mu}$. The two notations $\mathbf{e}$ and $=\mathbf{e}$ denote same entities and can be said to be synonymic denotations, but each of synonyms has its meaning - this is the reason for existence of many synonyms with same values (denotata). The format $=\mathbf{e}$ has the meaning we assigned to it - "the identity of $\mathbf{e}^{\prime \prime}$. Such a property of names (or "denotations") will become clearer when we will discuss later on the properties of names. Despite that such use of the equality sign is rather peculiar, the conceptually behind it has same fundamental character as the equality and it makes much sense in the A3 language.

Mind content is the "reality of mind". The Latin "rei“ stands for "things" or "objects" and "reification" is a term which denotes "making the reality" and it is used in Semantic Web to denote a method of reference to a statement. We will use this term with wider meaning and apply reification to any entities. Namely, by reification I mean „creating an image of an object in mind". 


\subsection{Reflection}

Mind content is synthesized to represent the Universe and, therefore, a piece of mind content is "in the image " of a piece of reality of the Universe. I will refer to this imagery by the term reflection. Reflection is a many-to-many correspondence between the identities in mind and the entities within universe which I can prove to be Galois connections - a structure widely used in algebra (Stewart, 1973) which has applications in various domains. Suppose that a piece $\mathbf{S}$ of mind content is in the image of a piece $\mathbf{O}$ of the Universe under an aspect. We will say that an aspect is due to a view of the agent, the conceptor. Aspect is on the side of the Universe and view is on the side of the conceptor. I will say both $\mathrm{S}$ and $\mathrm{O}$ to be universes, and to be specific on the "residence" of such universes - I will say S to be a subject universe, and $\mathrm{O}$ to be an object universe. Notice that $\mathrm{O}$ may also be a piece of mind content this situation is specific for the process of introspection. Taking into account the introspection, we did not say $\mathrm{S}$ to be "inner universe“ and $\mathrm{O}$ - „outer universe“. Also, due to different views there can exist many representations in mind of the same piece of reality. Therefore, the two universes must be correlated this manner only with respect to a representation $\mathrm{R}$. In other words, we examined the universes above with respect to the object-subject relationship within the context of a concrete representation $\mathrm{R}$.

\subsection{Structure and form}

I said that the subject universe is "in the image" of the object universe at some representation. In order for this imaging to make sense, the representation must preserve the "structure" or the "form" of the object universe and confer it to the subject universe. While we will define the notions of "structure" or "form" for the mind content, the question what might be these notions when applied for the objects of matter remains a problem and this "to be in the image" does not make sense. In order for it to make sense, by a conceptual "inverse engineering" method, I will ascribe the "structure" or "form" of the inner universe to the outer universe, and declare this to be the "structure" and "form" of any universe.

On a more general note, I make distinction between the notions structure and form by involving an agent's two complementary aptitudes, operation and view. Namely, if an agent builds via operations, i.e. "constructs", an object, then I say the object to be a structure (to remember, notice that to "con-struct" is linguistically correlated with "structure"). If an object is viewed by an agent, then I say it to be a form. A form can be "structured“ in different manners, depending on the analysis done by an agent viewing the result of construction, so that we can say that a form has many structures. On the other hand, if an object is viewed by an agent with memory, who memorized the construction process, then such an object „has one structure“ or we say „is a structure“. After a structure is created, it can be viewed - a structure is a partial case of form.

The mind content obtained in result of the synthesis process is a structure, versus a form. Really, at each step of the synthesis process, one A3 operation is applied, and each A3 operation is "invertable" - the arguments of the operation can be restored from its result. We will apply to mind content or to the universes the word structure and to the objects inside them the word form. This conforms with our saying that an object has a form.

While the distinction between structure and form is rather subtle, these two notions are essentially different and, in practice, different terminology is associated with each. Only by taking into account this distinction I will be able to explain below the essence of atomifications. Sometimes, the notion of "building block" in a construction process is 
referred to as "atom". We will use the word atom only with respect to forms and we will introduce other terms for structures.

I will say the entities from which starts a construction process in a structure $S$ to be the initial entities of the structure $S$, and the entity last produced in construction process of $S$ to be the final entity in structure $S$. The initial entity and the final entity are notions correlated with a structure and specify the boundary "in depth" which I say to be scale dimension. Such specification of boundary "in depth" is missing in set theory. So, when we said that a set of the form $\{\{a\},\{a, b\}\}$ is said to be an ordered pair $(a, b)$, we did not define an object, but expressed one form through another form. According such definition the set $\{\{\{a, b\}\},\{\{a, b\}$, a)\} is also an ordered pair, but it has another form. Mathematicians don't have difficulties with this because they subconsciously are doing additional mental work. But our goal is to reflect any mental work through formal processing of mind content.

Before defining what is a universe obtained in result of multiple applications of the A3 operations, it is useful to first study the "pure universes" consisting of entities of only one type - the universe of aggregations, the universe of associations, the universe of atomifications. Each of these universes can be considered as part (in the sense of subset or subclass) of a larger universe where reside entities of any A3 type. We will say this last universe to be the Full Universe, where I borrowed the word "full" from "full OWL" - the variant of the OWL Semantic Web language meant to reflect the Description Logic in full. This variant of OWL is only mentioned in standards, but it remains unspecified. Since the A3 language to be specified by the end of this paper has an absolute minimum of primitives, and the choice of such primitives is based on fundamenta mathematicae reasons, I assume that the A3 language can serve as a "nucleus" of the "full OWL".

\section{The universe of aggregations}

The universe of aggregations is the residence of only aggregations or, to use physical terminology, this is a universe which has only one dimensionality - the space dimensionality. This universe extends the universe of discourse of any axiomatic set theory so that the conceptuality and terminology of set theory can be applied to aggregations. I have actually extended, where applicable, the set theoretic terminology to Universics, and will use it for the aggregations.

The term aggregation denoting the result of the aggregation operation has the same meaning as in IT, but the aggregation operation which produces this result is studied only in set theory, where it is named "set abstraction" and is reflected in the notation $\{x: \varphi(x)\}$, for a formula $\varphi(x)$ in the language of set theory. Obviously, when $\varphi(x)$ is true for any value of $x$, for example, when $\varphi(x)$ is $(x=x)$, then the aggregation coincides with the universe of discourse of set theory.

The use of the term aggregation for set, is motivated by actually treating the term aggregation as wider than the term set. According classic axiomatic set theories, a set is defined by its elements so that two sets with same elements coincide due to the extensionality axiom. The meaning of this axiom is that a set has exactly one identity. I do not postulate the extensionality axiom for the universe of aggregations - actually, I don't postulate any axioms because axioms have to do with discourse, and a universe is a presentation framework. Therefore, in the universe of aggregations, two aggregations might be equal 
while their identities be different. One aggregation may have different identities at different times - a multitude of identities. But at one time an aggregation has exactly one identity.

We can define aggregations in terms of sets the following manner - an aggregation is a set together with an entity on the role of its identity. For all these reasons, we can say aggregations to be multi-identity sets, or multi-sets. Generally, by "multi-set" they refer to "simple", i.e., one identity set, the elements of which can be multi-identity. But if the quality of multi-identity is attributed to all the multi-sets in a universe of multi-sets, then it should apply not only to the elements of a multi-set, but also to the multi-set itself.

\subsection{Representation of aggregations in mind}

I will denote aggregations by using the regular set-theoretic notations for denoting sets and classes, which utilize the braces - "\{“ and ", $\}^{\prime \prime}$. So, the aggregation of the entities $a_{1}, \ldots, a_{n}$ will be denoted by $\left\{a_{1}, \ldots, a_{n}\right\}$. Notice, that in this denotation, the comma can be treated as an operation. I will say this operation to be assembly operation, because it actually can serve for the "element-wise" assembling a finite set out of it elements, and, since I have just introduced a new term, I will refer to its etymology, which is the French word "ensemble“, "togetherness" which served as the original name in French for a "set", introduced by the founder of "set theory“", Georg Cantor.

The assembly operation is commutative, associative and idempotent, where the last property means that for any $\mathbf{e}$ and $\mathbf{f}$ the following equality is true: $\{\mathbf{e}, \mathbf{e}\}=\{\mathbf{e}\}$. In this paper, $\mathrm{I}$ will not focus on this operation - I needed to mention about the assembly operation only for the purpose of making the notations of the A3 language precise, so that the comma is used only in notations where its properties are expected and to avoid its use in the contexts where such properties are not intended (like in denotation of associations).

Because an aggregation of $\mathrm{n}$ elements $\left\{a_{1}, \ldots, a_{n}\right\}$ has an identity it must be represented in mind by $\mathrm{n}+1$ entities: $\mathrm{n}$ identities of the elements $a_{i}$ and the identity, of the aggregation itself. Where is represented the identity of the aggregation in the notation $\left\{a_{1}, \ldots, a_{n}\right\}$ ? Such a question, obviously, makes sense only for a conceptor, the mental space of which is paper on which are placed the inscriptions of such notations. We will regard the pair of braces ", $\{$ " and "," as the denotation of the aggregation's unity because it makes out of the elements $a_{1, \ldots,}, a_{n}$ a unit of data. All the denotation, i.e. this object presentation (see above what is an "object presentation“) is an identity of the aggregation.

\subsection{The aggregation as an abstraction}

The application of the operation of aggregation results in an aggregation as a structure $\left\{a_{1, \ldots,}\right.$ $\left.a_{n}\right\}$. What is or what are the arguments of this operation? The first idea that these are $a_{1}, \ldots, a_{n}$ is not correct, because with such a treatment we would need to have an infinite number of $\mathrm{n}$-ary aggregation operations. I am treating this as an operation of abstracting the set from the universe by selecting those elements which are in the focus of the conceptor. This can be also an explaination of the term "set abstraction“. Thus, the abstraction operation applies to the whole universe of discourse.

The operation of "set abstraction" in set theory is known to cause logical contradictions, and one of the methods to avoid them is to limit its application to the elements of another set $U$, which is reflected in the following type of notation: $S=\{x \in \mathrm{U}: \varphi\}$. 
But the universe of entities in mind at a certain moment of time is finite and this abstraction is unlikely to create contradictions. Anyway, I treat the set abstraction, or aggregation, without any limitations on its applicability, as a natural operation of mind, even when used in a manner which creates contradictions. This is because a human can reason contradictorily and build in mind "impossible geometric pictures" like those of Bosch, including for the purpose of denying their existence. Similarily to the natural intelligence, we must allow the conceptor to create contradictory concepts and we will place no limitation on how it applies the aggregation operation.

Each aggregation $U$ can be treated as a universe of discourse, if we limit our discourse to the aggregations which are elements of $U$. Specifically to avoid contradictions, mathematicians replaced the notation $\{x: \varphi(x)\}$ of the intuitive set theory by the notation $\{x \in U: \varphi(x)\}$. But the intuition behind this sometimes escapes the regular users, and in practice sometimes the intuitive set theory notation is used. In Universics, which can be treated as an extension of intuitive set theory and as a presentation framework, the notation $\{x \in U: \varphi(x)\}$ is rather useless, because it actually reminds you which is the universe of discourse. In Universics, in order to avoid contradictions it is enough to follow the principle ",in reasoning, don't get outside the universe of discourse". Such principle can be referred to as "factoring out the universe" which has the meaning of "bringing a factor outside the brackets" (in this case outside the braces of aggregation).

Alongside universes of presentation, Universics also alows to conduct discourse and deal with universes of discourse. For this purpose, alongside operations, we need relationships and we will take over from set theory the membership relationship. In set theory this relationship is denoted by $\varepsilon$ (epsylon) or, more often, by a special sign similar to $\boldsymbol{\epsilon}$. In order to reduce the non-ASCII notations, I prefer to use the reserved word in, used with same meaning in database SQL language, to denote the membership relationship. In sync with this is the fact that the result of an SQL query is a multi-set, i.e. an aggregation.

In order to avoid contradictions, classical axiomatizations of set theory demand from the membership relationship to be acyclic, i.e., it does not admit the chains $\mathbf{s}_{\mathbf{1}}$ in ... in $\mathbf{s}_{\mathbf{n}}$, where $\mathbf{s}_{\mathbf{1}}=\mathbf{s}_{\mathbf{n}}$. But there are reasons for admittance of cyclic membership. So, for example, the set $S$ of infinite sets is itself an infinite set, while "S in $S^{\prime \prime}$ is true. Such set has a good and natural intuitive meaning for practice and in the intuitive set theory. Therefore, I will not demand for aggregations the membership acyclicity property, and will show that cyclic membership is also admissible for so called open or, more customarily "open world", aggregations.

Similarily to set theory, we can allow other entities to reside in the universe of aggregations and say them to be non-aggregations, ur-elements or atoms. The word "non-aggregation“ is rather a definition than a term and "ur-element" is a german-latin word - a mixed formation un-welcome by linguistics, but also implying that it is necessarily an element (of an aggregation), which is not always the case. Therefore, I preferred the term atom. Unlike the universe of aggregations and atoms, in an arbitrary universe it is recommended to use the term atomification, because in Universics, atoms are regarded as obtained via the operation of atomification.

Additionally the notion of set, there are also other notions generalized by the term aggregation. These are the notion of set theoretic class - an object obtained in set theory via the abstraction denoted by $\{x: \varphi(x)\}$ (not by $\{x \in \mathrm{U}: \varphi\}$ ). The notions class, container and collection of Semantic Web are also certain types of aggregations, which require separate treatment and are not in the focus of this paper. The only feature of these entities worth 
mentioning here is that in Semantic Web all are treated as multi-identity entities, and thus, fall under the incidence of aggregations.

\subsection{Graphical representation of aggregations}

The aggregations can be represented graphically as multi-hypergraphs. The multitude of identities of an aggregation is represented by multiple closed contours surrounding the elements. "To be immediately inside" is to be treated as "to be an element". Therefore, the multiple contours due to multitude of identities must intersect each other as in the Figure 3 below. The empty aggregations are represented as contours surrounding nothing. If there are atoms alongside aggregation, the atoms are represented by black pictures - here these are black small dots.

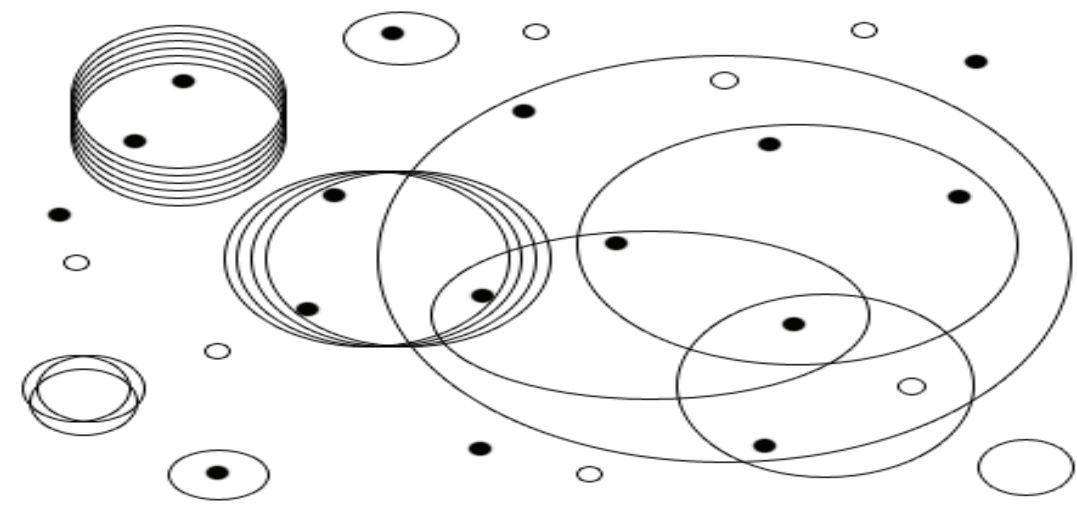

Fig. 3. A multi-hyper graph of aggregations

Notice, that the graphical representation method introduced above is rather limited. The main difficulty is with representing the complement of an aggregation. To fix this, we could introduce orientation to the contours. We can consider that the current graphical representation method is the method with oriented countours where, by default, the orientation is towards "the inside" and, therefore, it is not indicated.

Another difficulty appears when we want to enclose into a countour distant images separated by images which we don't want to inclose. Then the contour becomes so convoluted that the graph is more confusive than illustrative. This situation could be overcome by coloring the graphic elements, i.e., by using poly-chromatic multi-graphs.

The difficulties above hindered the use of hypergraphs, say nothing of poly-chromatic multigraphs, in Semantic Web, where they use only simple oriented (directed) graphs. This also hinders wide deployment of Semantic Web to deliver its methods, democratically, to biologists, chemists and other non-technical people.

\subsection{Operational set theory}

Because, by agent it is common to refer to an animate entity capable of action, and action consists of separate operations done by the agent, we could characterize an agent-oriented set theory as "operational" versus the usual "relational". An operational set theory must be based on primitive operations, versus primitive relations (of membership and of being an atom) of a 
classical set theory. The idea of "operational set theory" goes back to the "intuitive" or "naive" set theory developed in late 19th century. In this "theory", the sets, same as the axioms of an axiomatic set theory, are represented in mind as "mind content "taking the place of statements about them. Therefore such a "theory“ can do without axiomatization. In other words, the "intuitive set theory" is more of a "presentation framework" than a theory. The "intuitive set theory" was replaced by axiomatic set theories (many such theories) in order to avoid contradictions which appeared in the early 20th century, but in practice it remains up to day a useful framework without raising any contradictions. Moreover, in practice, they rarely make reference to any axiomatization and one reason for this is the multitude of existing axiomatizations.

As a formal framework, Universics is an agent-oriented operational and presentational framework and it can be considered a continuation of "naive" set theory. The certainty that Universics is consistent, i.e. that it lacks contradictions, is provided by the multitude of universes of discourse which ensures that by keeping within one universe of discourse, you cannot obtain contradictions. All the contraditions found in set theory are caused by unconsiously getting outside of the unique universe of discourse. True, Universics itself also has a universe of discourse, the entities of which are universes and, probably, contradictory constructs can be designed. But, if the status of meta-discipline of Universics is observed, and it is used only to discuss about universes of discourse of other disciplines, then such contradictions are highly improbabe.

\section{The universe of associations}

Associations are multi-identity ordered pairs. Applying association operation multiple times is a specialty of the left hemisphere of a conceptor - a component which can be said to be a "time machine".

I will denote an association by $(a: b)$ - a denotation which differs from denotation $(a, b)$ of an ordered pair used in set theory. As I explained above, comma denotes the assembly operator which has properties (commutativity, associativity, idemponence) different from those expected from a separator for association. Thus, the separator sign (:) widely used in many domains, including IT, will be also used for separation in denotation of an association. The notation $(a: b)$ is taken over to the A3 language as one of its primitive expressions where it has the meaning of qualification by the name $a$ of the name $b$. Such notation conforms with the practice of using the colon (:) in Semantic Web languages to qualify a name by a namespace.

I am treating the notion of association as orthogonal to the notion of aggregation, and this refers both to operations and the resulting entities. The reasons for such treatment are to avoid the difficulties encountered by set theory related to the notion of ordered pair and to enable their proper handling by an agent. In set theory, the ordered pair of two entities (sets or atoms) $a$ and $b$ (in this order) is denoted by $(a, b)$ and is defined as a set in different manners, where the best known definition is that of Kuratowski: $(a, b)=\{\{a\},\{a, b\}\}$. The only requirement from any such definition is that for any entities for any entities $a, b, a^{\prime}, b^{\prime},(a, b)$ $=\left(a^{\prime}, b^{\prime}\right)$, if and only if $\mathrm{a}=\mathrm{a}^{\prime}$ and $\mathrm{b}=\mathrm{b}^{\prime}$. Various authors provided a large number of definitions of the ordered pair, each with its merits and drawbacks. Actually, the notion of ordered pair can be defined by an infinite number of non-equivalent definitions, and this is one reason, why the property above should be treated as pertaining to entities of a type 
different from sets, and their formation - to an operation different from that of formation of sets. This entity type and operation is the association.

Defining an ordered pair as a special type of set was dictated by the necessity to avoid introduction of an extra sort of entities different from the sort set (and different from the sort atom, if the theory is set theory with atoms). Such a definition is good only for theory, but for an agent viewing the universe of sets and ordered pairs defined as a special type of sets, such definition makes impossible the recognition of the entity type. Namely, if an agent views the set $\{\{a\},\{a, b\}\}$, it does not know, whether to consider this an ordered pair or a set which, coincidentally, has same form. Also, an author who meant $\{\{a\},\{a, b\}\}$ to be a set, cannot encrypt this information in this denotation and would have to add it in words like „where by $\{\{a\},\{a, b\}\}$ I mean a set and not an ordered pair". For a virtual machine there can appear also other problems due to incomplete encryption of information. For all these reasons I regard the notion of ordered pair as orthogonal to the notion of set.

In set theory, the notion of $n$-tuple is defined by induction: $\left(a_{1}, a_{2} \ldots, a_{n}\right)=\left(a_{1},\left(a_{2} \ldots, a_{n}\right)\right)$.

This definition is interesting mostly for mathematics, but Semantic Web uses oriented (directed) graphs which can be represented by various superpositions of associations. This a reason, why we will introduce no default aggreement like "association to the right (left)" similar to set theory or other special domains. Various structures can be represented by different arrangement of parentheses and this is the "modeling methodology" of some programming languages like LISP. Probably, it is exactly due to the richness of structures obtained by various arrangement of parantheses that the language LISP is so powerful as to serve for the AI modeling.

The most ancient use of round parentheses is indication of the order of application of various operations in a formal expression by enclosing in round parentheses the results of some applications of various operations. We will say such use of round parentheses to be formal association, because sometimes such grouping is said to be "associating". We need to separate the formal use of round parentheses and their use as denotation of an association ( $a$ $: b$ ), where the round parantheses (together with colon) make part of the syntax of the A3 language to be specified later. We will agree to always use the round parantheses of the A3 language syntax except, maybe, the "external“ ones of a complete expression. With such agreement, the formal association cannot create any confusion.

Given an association $A=(a, b)$ we will denote $a$ by source $(A)$ and $b$ by target $(A)$. Notice that due to formal convention above, the last two notations can have also the form "source $A^{\text {" }}$ and "target $B^{\prime \prime}$. In the universe of associations source and target are operations which interact with the association operation according the following correlations:

$$
\text { source }(a, b)=\mathrm{a} \text {, target }(a, b)=b, c=(\text { source } c \text {, target } c) .(1)
$$

The term member (or element) pertains to the language of set theory, and by extension - to the language about the universe of aggregations. I will adopt this term for associations as universe two association memberships. Namely, we will say both the source and the target of an association to be elements or members of the association.

Same as the universe of aggregations, the universe of associations can also contain atoms. Associations can be graphically represented by generalized multi-orgraphs. Oriented graphs, or simply, orgraphs are also said to be directed graphs. Such graphs are used in the RDF standard of Semantic Web. In Universics, to be able to graphically represent all types of 
associations, we need to use multi-orgraphs, i.e. graphs with multiple directed arcs. Moreover, since there are no limitations on application of the association operation, an arc also may connect nodes with arcs or only arcs as in the Figure 3.

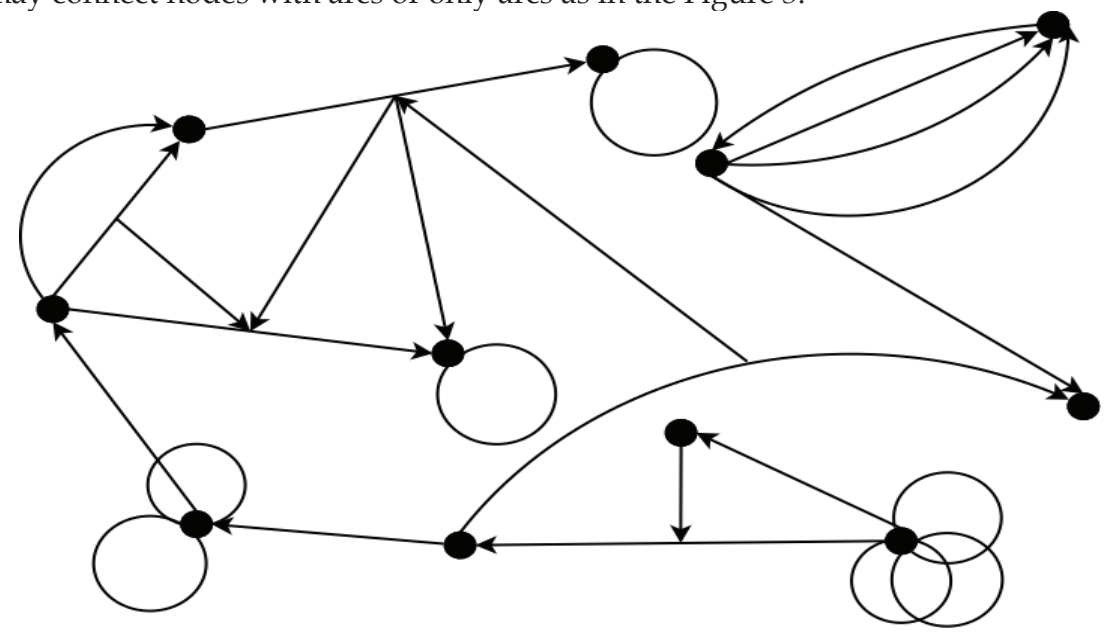

Fig. 4. Graphical representation of associations

\section{The universe of atomifications}

The operation and entity type called atomification are a new operation and entity type introduced in mathematics by Universics. The atomification operation can be treated as the operation of making a "whole" and it can be attributed to mereology - a term coming from Gr. "meros", "part". Mereology goes back to the beginning of the 20th century when the mathematicians were buiding a strict foundation for mathematics and most of them we focused on set theory as such a foundation. The Polish mathematician Lesniewski proposed mereology as an alternative foundation, but since only a restricted part of public could read his works in Polish, mereology is better known from the publications of the American mathematician Goodman, who also authored a monography (Goodman, 1951), where he tried to place all mathematics on the basis of this science.

Mereology was initially regarded as the science about the "part-whole" relationship, but later it was found that representing all the discipline of research was actually focused on the relationship between parts, which was then said to be parthood relationship, while the relation between parts and the whole remained an open problem. The aspect of "wholeness" does not seem to have been fully clarified up to our days. The atomification operation of Universics seems to clarify the aspect of "whole“ from the point of view of structure.

Many mathematicians regarded mereology as a foundation for mathematics better than set theory and the competition between the two frameworks continued up to 1970's, when the "truce“ was established with the aggrement that both approaches have good methods and should complement each other. Today, building a natural "bridge“ between the two approaches, or even better, integrating the two approaches, is important for Semantic Web where, on one hand, many researchers develop mereology ontologies needed in various domains and, on the other hand, the semantics of Semantic Web standards is formulated in 
the language of set theory. As a formal framework, Universics integrates set theory and mereology into one discipline. I will now explain informally how Universics does this.

Set theory is regarded by most mathematicians as the best framework for representing structures. The mereological parthood relationship, which at a certain time in the past was regarded as all the mereology, is represented in the universe of sets as two simple relationships - the membership relationship and the sub-set relationship.

The simplicity of set theoretic methods and the illusion that all of the mereology is comprised in set theory, determined that set theory became the main formalization framework and mereology remained as just as an alternative approach. But the notion of ,whole' is very important for any approach, say nothing about a holistic approach, and set theoretic methods cannot deal with this notion. On the other hand, mereology, even though did not offer a "final solution" regarding part-whole relationship, developed a number of good mathematical and conceptual methods to treat this relationship.

Based on the reasons formulated above, Universics takes from each discipline their strength: from set theory focused on structures - the formalization of structures, and from mereology focused on forms - the notion of a whole, for which it introduces the atomification operation of making a whole. The difference between structures and forms has been explained in section 3.2.

Now I will proceed to atomifications, and will start with their rudiments encountered in set theory and said to be atoms. In set theory, an atom is an entity within the universe of discourse which is not a set. Thus, set theory regards the two notions, set and atom, as conceptually orthogonal, i.e. irreducible to one another. To conform to Greek etymology, according which "a-tom" means "un-cut", the word atom must be treated as an entity indivisible under certain aspect. I regard such aspect of an entity as dependent on the agent's view. Namely, I treat the property of being an atom, the atomicity, as a kind of opacity of the view of this entity. In the language of behavior of a conceptor, the opacity can be expressed as interdiction to zoom into the structure of an entity. Thus, the atomicity expresses the agent's intention to ignore the structure of the entity.

The property to be an atom, the atomicity, is relative and is correlated to an agent or, more precisely, to an agent's view. This conforms with the relativity of what is said to be "indivisibility“. So, in material world, the matter might be infinitely divisible and it is the agent view which determines whether something is or it is not divisible. In order to discriminate between the notion of atom described above, from other notions, say, from the notion of atom introduced in physics by Democrites, we could refer to our atom as "conceptual atom". But in this paper, we deal only with concepts and the qualification "conceptual" is superfluous.

I treat the notion of atomification as a common name (and concept) for the atoms and the "whole". The reason for such treatment is that a structure as a whole can also serve as an atom in another structure. To put it in other words, the notion of atom and the notion whole are correlated with a structure, and while an entity $\mathrm{E}$ is an atom of a structure $\mathrm{T}$, the entity $\mathrm{E}$ can also be inself a structure with its atoms, or to reflect the logic in language - it can be "the whole of the another structure". This logic shows that "the whole" is an attribute (more precisely - the value of the attribute) of a structure and, thus, is different from the structure. Because by whole we are used to refer to the structure itself, I will use another name for this entity - unity of the structure. To sum up, a structure has atoms, maybe many atoms, and it has one unity, and I refer to both these types of entities as atomifications. 


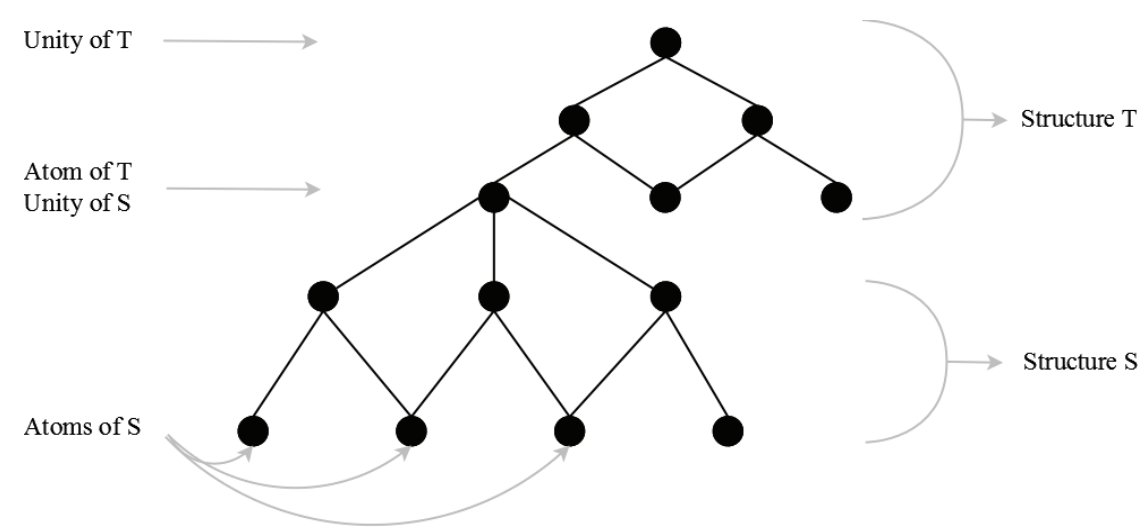

Fig. 5. Atoms and unities

The intuition behind these notions can be explained by reference to the physical matter which has a multi-layered organization in granularity levels: subatomic particles, physical atoms, planets and stars, galaxies, or to the world of biology: biomolecules, cells, animals, populations, etc. I say any of the "grains" at a certain level of granularity to be an atomification and regard it as obtained by an operation also said to be atomification.

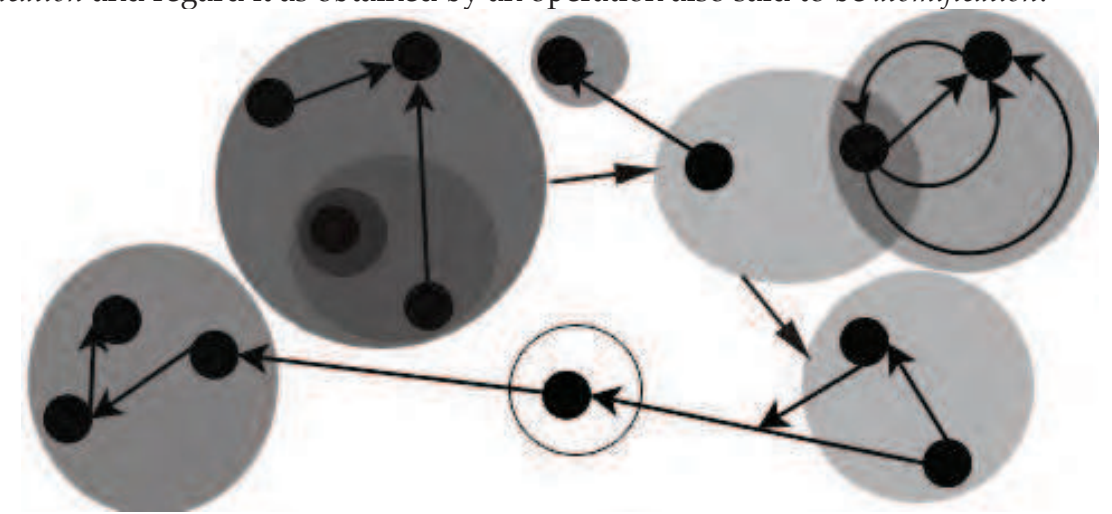

Fig. 6. Graphical representation of atomifications

A universe of atomifications is a universe governed by the part-whole relationship. Atomicity is treated by the conceptor as opacity of its view. By atomifications we mean both unity "making the whole" for a structure and the atoms of a structure. Therefore, to graphically represent a universe of aggregations, we need to draw atoms with different degrees of transparency, which still allow viewing inside an entity. Such "view control“ can be generalized in order to reflect many concepts in programming.

\section{The form and structure of a universe}

At this point we have all the conceptuality and terminology ready to formulate the notion of universe. It is easier to define the notion of universe after the notion of body, even though the 
usual meaning of body as a „body in the Universe“ implies that an inverse approach would be more appropriate. I am also using the linguistic term corpus (pl. corpora) for a body of knowledge, and I will treat these two notions structurally and formally the same. Therefore, body and corpus can be defined in one definition.

A body (or corpus) $\mathrm{B}$ is a tuple (A, $\left.\mathrm{A}_{1}, \mathrm{~A}_{2}, \mathrm{~A}_{3}\right)$, where $\mathrm{A}$ is a set said to be the foundation of the body $B$, its elements are said to be entities of the body $B$, and the other components are defined as below:

1. $\mathrm{A}_{1}$ is a 1-ary relationship (property) of "being an atom", or atomicity property, and an entity with this property - an atom,

2. $\mathrm{A}_{2}$ is a 2-ary relationship over $\mathrm{A}$, said to be aggregation relationship and, for any $(i, x)$ in $\mathrm{A}_{2}, i$ is said to be (aggregation) unity of the set $\left\{x \in \mathrm{A} \mid(i, x) \in \mathrm{A}_{2}\right\}$ and $\mathrm{x}$ is an element of this set,

3. $A_{3}$ is a 3-ary relationship over $A$ said to be association relationship and, for any $(i, x, y)$ in $A_{3}, i$ is said to be the (association) unity of the ordered pair $(x, y)$.

An argument $\mathbf{i}$ of a n-ary relationship $\mathrm{R}$ is said to be primary key, if the following statement is true for any values of its variables:

$$
\text { IF } \left.\left.\left(R\left(x_{1}, \ldots, x_{n}\right) \text { AND R( } y_{1}, \ldots, y_{n}\right) \text { AND }\left(x_{i}=y_{i}\right)\right) \text { THEN (FOR ANY } j\right)\left(x_{j}=y_{j}\right) \text {. }
$$

A universe is said to be a body whose identities are primary keys. Examples of universes are the universes of discourse of (axiomatic) set theories.

The knowledge representation method of Universics is obtained by assigning algebraic meaning to the statement "A body of knowledge is a homomorphic image of a body in the Universe". The notion of homomorphic image formalizes the subject-object relationship for universes as it was treated above in the section 3.5.

A homomorphism of a body $B=\left(A, A_{1}, A_{2}, A_{3}\right)$ into the body $B^{\prime}=\left(A^{\prime}, A^{\prime}{ }_{1}, A^{\prime}{ }_{2}, A^{\prime}{ }_{3}\right)$ is a triple $\left(B, h, B^{\prime}\right)$, where $\mathbf{h}$ is a function from $A$ to $A^{\prime}$, such that $\mathbf{h}$ preserves each of the relationships $A_{n}$, i.e., for $n=1,2,3$, and $a\left(x_{1}, \ldots x_{n}\right)$ in $A_{n}, h\left(x_{1}, \ldots x_{n}\right)=\left(h\left(x_{1},\right), \ldots, h\left(x_{n}\right)\right)$. This is a very compact definition which allows to generalize the notion of open function from topology, homomorphism from algebra, and introduces the notion of preservation of discreteness (vs continuality). To discover the homomorphism behavior in each of the three universal dimensions, this definition needs to be examined in detail for each value of $\mathbf{n}$.

\section{The $\mathrm{A} 3$ language}

The vocabulary of this language consists of two sorts of atoms

- An infinite set of main symbols,

- Nine auxiliary symbols:

○ Aggregation symbols " $\{$ ", " $\}$ " and comma ","

○ Association symbols "(", ")" and colon ":"

- Atomification symbols "[", "]"

- Equality symbol "="

In software, we can require the main symbols to be any string of Unicode characters except the auxiliary symbols. The expressions have different meaning and are named differently depending on whether they are used for denotation or for discourse. The expressions of A3 
language are defined by the rules below, where the names of expressions reflect their semantics: aggregation, association, identity.

1. If $a$ is a main symbol, then $a$ is an expression said to be an atom (denotation), or individual (discourse);

If $a$ is an expression, then $[a]$ is an expression said to atomification of $a$ (denotation) or reference to expression $a$ (discourse);

2. If $a 1, \ldots$, an are expressions, then $\{a 1, \ldots, a n\}$ is an expression called aggregation of $a 1, \ldots$, an (denotation) correlation with correlates $a 1, \ldots$, an (discourse)

3. If $a$ and $b$ are expressions, then $(a: b)$ is expression said to be the association of $a$ to $b$ (denotation) or qualification of $b$ by $a$ (discourse);

4. If $a$ is an expression, then $=a$ is an expression said to be identity of $a$.

Since the use of the expressions of A3 language for denotation of structures was already explained, I will discuss only about its use in discourse. Notice, that a discourse is a body of knowledge, and the structure of a discourse was defined in section 7 .

In Semantic Web the discourse is formulated in triples. You can read the triple $<a b c>$ like this: "a has the property b with value c". The "synonymic" expression of the "A3 language" is $((\mathrm{b}: \mathrm{c}): \mathrm{a})$. Also, you may use any n-tuples in discourse, in particular, the pair $(\mathrm{a}: \mathrm{b})$ expresses the same as the triple <a rdf:type b>.

In Semantic Web, in order to make reference to a set of triples (graph), you have to write complex XML code. In A3, you enclose a set of expressions ((b:c):a) between braces and qualify this construct. You may also represent this structurally as in programming languages - closing brace under opening brace.

In languages of Semantic Web, you cannot name a triple or a discourse other than by wrapping them in complex XML code. In A3 language you just enclose the text between square brakets.

The statement that two names are equal (synonymy) is represented in OWL by the triple $<a$ owl:sameAs $b>-$ in A3 it is represented as $a:=b$ (" $a$ qualifies the identity of $b$ ").

In Semantic Web, there is a special technique and are used long expressions to express an nary relationship. In A3 language, you just name the correlates of the relationship and enclose them between braces. You can also qualify this expression to obtain different relationships with same signature of names for the correlates.

Blank nodes of Semantic Web are just "temporary names" which can be changed by other names, and in A3 language, you just precede such names with an equality sign with the meaning "equal to any other name". To comply with notation of blank node of Semantic Web, a special name denoted undersore "_" can be added to A3 language as "syntactic sugar", so that for any name $a, " \_: a$ " is defined as " $=a$ ".

In Semantic Web there is no possibility to express syntactic correlations between expressions - in particular, you cannot define one expression through other expressions. In A3, you just enclose such expressions between square brakets. Say, to denote the expression " $x+y / 2$ " by " $f(x, y)$ ", you just write $[f(x, y)]:=[x+y / 2]$.

\section{Aknoledgements}

I would like to express my gratitude to my friend Bettin Mironov, a great physicist, who managed to reduce the physical laws to interaction of waves (Mironov, 2007). The long 
discussions with him provided "physical intuition" which helped in my mathematical research .

I am also greatful to my colleague Grigoriy Kunetskiy whose "difficulties" in implementing Universics in software and sharp "feeling of truth" helped so much to formulate correct definitions.

\section{References}

Church, A. (1941). The Theory of Lambda Conversion, Princeton University Press, (1941).

Chen, P. (1976), The entity-relationship model - towards a unified view of data, ACM Transactions on Database Systems (TODS), v.1. n.1, (March 1976), p.9-36

Codd, E. (1970), A relational Model of Data for Large Shared Data Banks. Communications of the ACM, Vol.13, No.7, (June 1970), pp.377-387

Cohen, P. (1963). "The Independence of the Continuum Hypothesis". Proceedings of theNational Academy of Sciences of the United States of America 50 (6), (December 1963), pp. 1143-1148.

Cohen, P. (1964). The Independence of the Continuum Hypothesis, II". Proceedings of the National Academy of Sciences of the United States of America 51 (1), (January, 1964), pp. 105-110.

Drugus, I. (1982). Forest superintuitionistic logics. Doklady Academii Nauk SSSR. (February, 1982). Volume 262. Nr 3.

Drugus, I. (2007), A Wholebrain approach to the Web. In: Proceedings of the "Web Intelligence - Intelligent Agent Technology Conference", Silicon Valley, (November, 2007).

Drugus, I. (2009), Universics - a structural framework for knowledge representation. Knowledge Engineering: Principles and Techniques Conference. Studia Universitatis, Babes-Bolyai, (July, 2009), pp. 115-118

Drugus, I. (2009), Universics: an Approach to Knowledge based on Set theory. In: Knowledge Engineering Principles and Techniques, Selected Extended Papers (Cluj-Napoca, Romania, July 2-4, 2009), Presa Universitara Clujeana (October, 2009), pp. 193-200.

Goodman, N (1951), The structure of appearence, Harvard University Press - Cambridge Massachusetts. (1951) Stewart, I. (1973). Galois Theory, Chapman and Hall, (1973)

Mironov, B (2007), Mechanisms of electromagnetic and gravitational fields, (2007)

Turing, A.M. (1936/7). On computable numbers, with an application to the Entscheidungs problem, Proceedings of the London Mathematical Society 42, pp. 230-265. (1936/7)

Turing, A.M. (1937). Computability and $\lambda$-definability, Journal of Symbolic Logic 2, pp. 153$163(1937)$ 


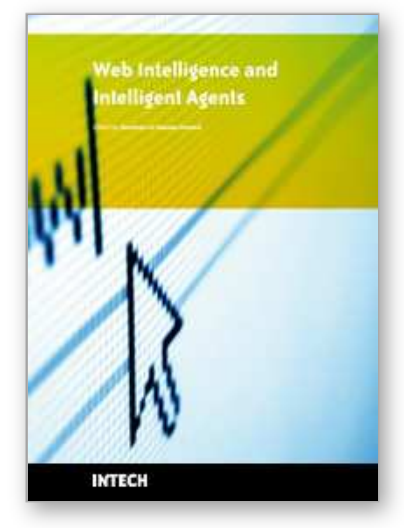

\section{Web Intelligence and Intelligent Agents \\ Edited by Zeeshan-UI-Hassan Usmani}

ISBN 978-953-7619-85-5

Hard cover, 486 pages

Publisher InTech

Published online 01, March, 2010

Published in print edition March, 2010

This book presents a unique and diversified collection of research work ranging from controlling the activities in virtual world to optimization of productivity in games, from collaborative recommendations to populate an open computational environment with autonomous hypothetical reasoning, and from dynamic health portal to measuring information quality, correctness, and readability from the web.

\section{How to reference}

In order to correctly reference this scholarly work, feel free to copy and paste the following:

Ioachim Drugus (2010). Universics - a Common Formalization Framework for Brain Informatics and Semantic Web, Web Intelligence and Intelligent Agents, Zeeshan-Ul-Hassan Usmani (Ed.), ISBN: 978-953-7619-85-5, InTech, Available from: http://www.intechopen.com/books/web-intelligence-and-intelligent-agents/universics-acommon-formalization-framework-for-brain-informatics-and-semantic-web

\section{INTECH}

open science | open minds

\section{InTech Europe}

University Campus STeP Ri

Slavka Krautzeka 83/A

51000 Rijeka, Croatia

Phone: +385 (51) 770447

Fax: +385 (51) 686166

www.intechopen.com

\section{InTech China}

Unit 405, Office Block, Hotel Equatorial Shanghai

No.65, Yan An Road (West), Shanghai, 200040, China

中国上海市延安西路65号上海国际贵都大饭店办公楼 405 单元

Phone: +86-21-62489820

Fax: +86-21-62489821 
(C) 2010 The Author(s). Licensee IntechOpen. This chapter is distributed under the terms of the Creative Commons Attribution-NonCommercialShareAlike-3.0 License, which permits use, distribution and reproduction for non-commercial purposes, provided the original is properly cited and derivative works building on this content are distributed under the same license. 